\title{
TIME DELAY MEASUREMENTS FOR THE CLUSTER-LENSED SEXTUPLE QUASAR SDSS J2222+2745*
}

\author{
H. Dahle ${ }^{1}$, M. D. Gladders ${ }^{2,3}$, K. Sharon ${ }^{4}$, M. B. Bayliss ${ }^{5,6}$, and J. R. Rigby ${ }^{7}$ \\ ${ }^{1}$ Institute of Theoretical Astrophysics, University of Oslo, P.O. Box 1029, Blindern, NO-0315 Oslo, Norway; hdahle@astro.uio.no \\ ${ }^{2}$ Department of Astronomy \& Astrophysics, The University of Chicago, 5640 S. Ellis Avenue, Chicago, IL 60637, USA \\ ${ }^{3}$ Kavli Institute for Cosmological Physics at the University of Chicago, USA \\ ${ }^{4}$ Department of Astronomy, University of Michigan, 500 Church Street, Ann Arbor, MI 48109, USA \\ 5 Harvard-Smithsonian Center for Astrophysics, 60 Garden Street, Cambridge, MA 02138, USA \\ ${ }^{6}$ Department of Physics, Harvard University, 17 Oxford Street, Cambridge, MA 02138, USA \\ ${ }^{7}$ Observational Cosmology Lab, NASA Goddard Space Flight Center, Greenbelt, MD 20771, USA \\ Received 2015 May 22; accepted 2015 September 30; published 2015 October 29
}

\begin{abstract}
We report first results from an ongoing monitoring campaign to measure time delays between the six images of the quasar SDSS J2222+2745, gravitationally lensed by a galaxy cluster. The time delay between A and B, the two most highly magnified images, is measured to be $\tau_{\mathrm{AB}}=47.7 \pm 6.0$ days $(95 \%$ confidence interval), consistent with previous model predictions for this lens system. The strong intrinsic variability of the quasar also allows us to derive a time delay value of $\tau_{\mathrm{CA}}=722 \pm 24$ days between image $\mathrm{C}$ and $\mathrm{A}$, in spite of modest overlap between their light curves in the current data set. Image $\mathrm{C}$, which is predicted to lead all the other lensed quasar images, has undergone a sharp, monotonic flux increase of $60 \%-75 \%$ during 2014. A corresponding brightening is firmly predicted to occur in images A and B during 2016. The amplitude of this rise indicates that time delays involving all six known images in this system, including those of the demagnified central images D-F, will be obtainable from further ground-based monitoring of this system during the next few years.
\end{abstract}

Key words: galaxies: clusters: general - gravitational lensing: strong - quasars: individual (SDSS J2222+2745)

\section{INTRODUCTION}

Quasars gravitationally lensed into multiple images by foreground clusters of galaxies are exceptionally rare. The two first reported cases were SDSS J1004+4112 (Inada et al. 2003) and SDSS J1029+2623 (Inada et al. 2006). A third case, SDSS J2222+2745, was discovered by Dahle et al. (2013), displaying six images (of which five were spectroscopically confirmed) of a quasar at redshift $z_{s}=2.82$, with a maximum separation of 15 .' 1 , lensed by a foreground cluster at $z_{s}=0.49$. In contrast, the Master Lens Database of known gravitational lens systems (Moustakas et al. 2012) lists $~ 120$ known galaxyscale quasar lenses.

Refsdal (1964) showed how the relative time delays measured in multiply lensed quasar images can be used to measure the Hubble parameter $H_{0}$. In addition, time delay measurements can probe the underlying cosmological model (Refsdal 1966), yielding dark energy constraints that are complementary to other such probes (Linder 2011; Treu et al. 2013; Sereno \& Paraficz 2014). Time delay measurements of individual systems are subject to a "cosmic variance" of several percent, caused by the mass fluctuations of intervening structure along the line of sight (e.g., Bar-Kana 1996; Wambsganss et al. 2005). Aside from the cosmographical applications of time delays, they also probe the surface density distribution of the lens, over the range of radii at which images

\footnotetext{
* Based on observations made with the Nordic Optical Telescope, operated on the island of La Palma jointly by Denmark, Finland, Iceland, Norway, and Sweden, in the Spanish Observatorio del Roque de los Muchachos of the Instituto de Astrofisica de Canarias, and including observations obtained at the Gemini Observatory, which is operated by the Association of Universities for Research in Astronomy, Inc., under a cooperative agreement with the NSF on behalf of the Gemini partnership: the National Science Foundation (United States), the National Research Council (Canada), CONICYT (Chile), the Australian Research Council (Australia), Ministério da Ciência, Tecnologia e Inovação (Brazil) and Ministerio de Ciencia, Tecnología e Innovación Productiva (Argentina).
}

are seen and time delays are measured. A unique property of SDSS J2222+2745 is the availability, at small clustercentric radii, of three fainter images (D-F) that are predicted to be demagnified, according to the six-image model presented by Dahle et al. (2013). These three images are still sufficiently bright to have their time delays measured from ground-based data, given the level of quasar variability reported in this paper.

The longest time delays produced by cluster-scale lenses can be of the order of several years (Fohlmeister et al. 2008, 2013), rather than weeks or months in the case of galaxy-scale lenses. With typical monitoring cadence of a few days to a few weeks, and given the measured trend of quasar variability amplitude to increase as a function of time scale (at least up to time scales of several years; e.g., Vanden Berk et al. 2004), cluster-scale lenses may yield smaller fractional uncertainties on the time delays, making cosmic variance-limited time delay measurements easier to obtain than for galaxy-scale lenses. A further advantage of cluster-scale lenses is the availability of additional constraints on the lensing mass distribution from other multiply lensed background galaxies as exemplified by the Hubble Space Telescope (HST)-based studies of SDSS J1004+4112 (Sharon et al. 2005; Oguri 2010) and SDSS J1029+2623 (Oguri et al. 2013).

Measuring the time delays in wide-separation cluster-lensed quasars also enables three-dimensional studies of outflowing winds within the quasar itself by probing multiple sightlines, as shown by Misawa et al. (2014). In this case, the time delays need to be known in order to disentangle time variability of absorption profiles in the quasar image spectra from genuine differences between the sightlines.

Finally, knowing the time delay is crucial for advance planning of monitoring with increased frequency in anticipation of predicted strong flux changes, e.g., to measure the time delays of the faintest images in the system or to conduct reverberation mapping studies to measure the mass of the black 
hole that powers the quasar (Blandford \& McKee 1982; Peterson 1993, 2014).

In this paper, we summarize results obtained from the first three years of an ongoing monitoring campaign of SDSS J2222+2745. In Section 2 we present the data set available so far, which is used to derive a value of the time delay $\tau_{\mathrm{AB}}$ in Section 3 and a value of the longer delay $\tau_{\mathrm{CA}}$ in Section 4. In Section 5, we summarize and discuss the results, including a strong rise in the flux of image C during 2014, and the resulting prospects for future studies of this system.

\section{DATA}

A monitoring campaign to measure time delays in SDSS J2222+2745 was initiated at the $2.56 \mathrm{~m}$ Nordic Optical Telescope (NOT) in 2012 October. The results in this paper are based on data from this ongoing campaign, with the most recent data obtained in 2015 August. The NOT monitoring program makes use of the Andalucia Faint Object Spectrograph and Camera (ALFOSC) instrument, which has a $2048^{2}$ pixel CCD detector with 32 bit intensity resolution and a pixel scale of 0 !' 188 pixel $^{-1}$. At each monitoring epoch, a set of either $3 \times 300 \mathrm{~s}$ or $3 \times 600 \mathrm{~s}$ exposures were obtained.

The annual visibility period of SDSS J2222+2745 extends from late April until early January. We chose the SDSS $g$-band for our monitoring campaign, in order to maximize the signalto-noise of the quasar images, relative to the galaxies in the $z=0.49$ lensing cluster on which the central D-F images are superposed. This band is well centered on the prominent Ly $\alpha$ line in the spectrum of the $z=2.82$ quasar, at an observed wavelength of $4640 \AA$.

In addition to the ALFOSC data, we include $g$-band data obtained as part of the original discovery of this lens (for further details, see Dahle et al. 2013), using the MOSaic CAmera (MOSCA) at NOT. MOSCA is a $2 \times 2$ mosaic of four $2048 \times 2048$ CCDs used in $2 \times 2$ binned mode, yielding a pixel scale of 0 "' 217 pixel $^{-1}$. MOSCA $g$-band imaging was obtained for three separate epochs, on 2011 September 24.93, 2012 September 12.96, and 2012 September 15.98 UT, respectively. We also include the original SDSS DR8 $g$-band photometry for this system, obtained on 2009 October 18.19 UT. Finally, this paper also includes recently acquired Gemini imaging of SDSS J2222+2745 (taken under program GN2015A-FT-16); these few images represent the beginning of a new observational campaign to measure time delays for the much fainter central D-F images. Those data were acquired in the $g$-band with Gemini North, using the GMOS instrument in imaging mode, on 2015 May 25.54, 26.52, and 2015 July 25.48 UT.

In this paper, we report photometric measurements of the three brightest (A-C) images of SDSS J2222 +2745. Unlike the fainter (D-F) images, these are all well separated from foreground objects and their fluxes can be reliably measured without modeling and removal of additional sources. In order to calibrate the observed fluxes, we use a set of five reference stars (S1-S5; see Figure 1). In order to minimize the effect of spatial variations of the point-spread function, these are chosen within a radius $1^{\prime}$ from the quasar images. In addition, the reference stars have blue colors similar to those of the quasar and do not have any nearby objects that may affect the flux estimate. Even during the best seeing (FWHM $\sim 0$ "' 5), these stars have measured peak fluxes $\lesssim 80 \mathrm{kADU}$, well within the linear regime of the ALFOSC detector, which extends up to

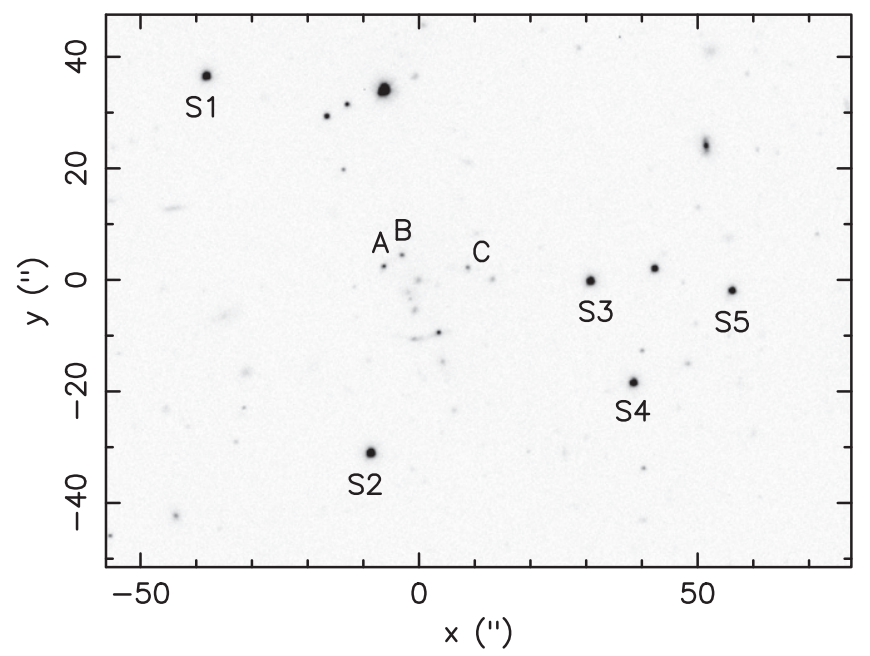

Figure 1. A single $600 \mathrm{~s}$ ALFOSC exposure, with the locations of the five comparison stars S1-S5 indicated.

$\sim 350-400 \mathrm{kADU}$. The photometric measurements in our data were all based on aperture photometry of the quasar images and reference stars, using the same aperture. An aperture correction was implicitly made by setting the magnitude zero point of each exposure such that the mean magnitude value of the ensemble of five reference stars equals their mean $g$-band magnitude value in the SDSS $(g=19.092)$. The accuracy of the time delay measurements reported in this paper is not dependent on a highly accurate absolute flux calibration, as long as the reference stars do not vary significantly in brightness. The validity of using $\mathrm{S} 1-\mathrm{S} 5$ as flux reference sources was tested by measuring the rms fluctuation in the magnitude offset of each star from the ensemble average, over the time span covered by our ALFOSC observations. The rms value of these fluctuations range between 0.0061 and $0.0095 \mathrm{mag}$. This is two orders of magnitude smaller than the amplitude of the flux variations measured in SDSS J2222 +2745 , as described below.

The ALFOSC imaging data were de-biased and flat fielded using standard techniques. Flat-fielding was typically performed using the median of a series of flat field frames of the twilight sky obtained during the same night as the photometric data. As the ALFOSC detector is cosmetically clean, the three individual exposures for each monitoring epoch were treated individually in our analysis rather than combined into a single frame.

The Gemini GMOS imaging data were de-biased and flat fielded, again using standard techniques, with appropriate and temporally adjacent master bias and flat field frames taken from the Gemini Science Archive. Each Gemini observation is the sum of six dithered $300 \mathrm{~s}$ integrations, stacked to eliminate cosmic rays. Care was taken to avoid improperly rejecting pixels in the cores of bright stars when stacking the dithered frames. Though images A-C are well measured in each GMOS sub-image, the thick red-sensitive CCDs in use on GMOSNorth are also excellent charged particle detectors, and the most robust measurements thus come from stacked images with good cosmic ray rejection.

The fluxes of SDSS J2222+2745 images A-C and reference stars S1-S5 were all measured within a fixed aperture of diameter 1".13, for the ALFOSC, MOSCA, and GMOS data. The choice of aperture scale was motivated by containing most 


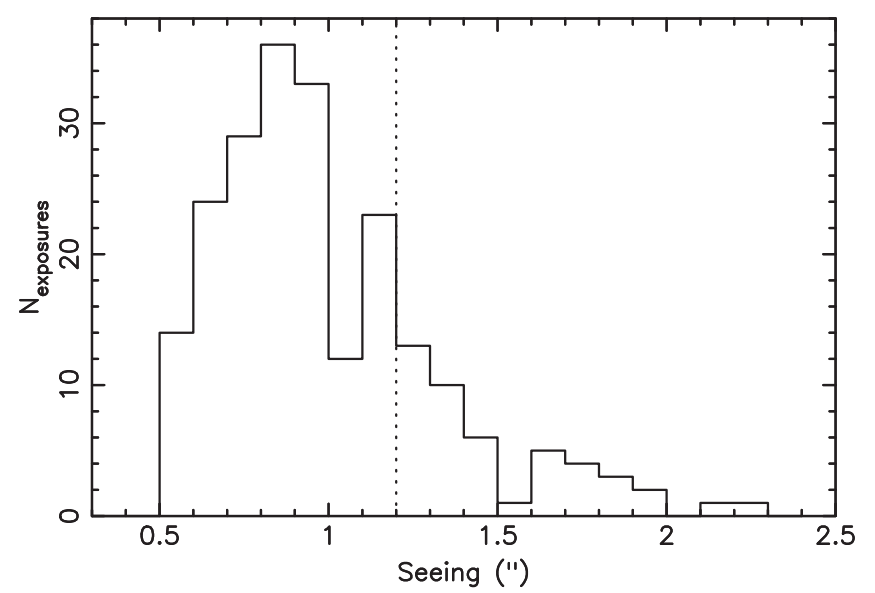

Figure 2. Histogram of seeing values measured in individual ALFOSC exposures. The dotted vertical line corresponds to the upper cutoff value for data used in our analysis.

of the flux while avoiding contamination from nearby sources. The effect on the time delay measurements of choosing an aperture a factor 2 smaller was tested, and found to produce changes in the time delay estimate within the uncertainties quoted below. The photometric uncertainty was determined by measuring the photometric scatter corresponding to the three individual ALFOSC exposures and fitting the result as a function of the signal-to-noise of the quasar images.

Figure 2 shows a histogram of seeing values measured in the individual ALFOSC monitoring exposures. In our further analysis, we excluded data points obtained when the FWHM seeing was worse than 1 !' 20 , and we also excluded data where the sky background level was very high, e.g., caused by moonlight or twilight. This left 42 distinct epochs of ALFOSC monitoring observations (not counting multiple exposures within the same night), with an average time span between observations of 14 days, during the annual visibility period of this target. The photometric measurements used in our further analysis are listed in Table 1.

Significant flux variability of the three brightest images is evident even from a cursory inspection of the imaging data: Figure 3 qualitatively illustrates the flux changes over a 3.2 year time span: in 2011 September, the A and B images were both obviously brighter than the C image. By 2014 November, the A and B components had both faded while approximately preserving the $\mathrm{A}: \mathrm{B}$ flux ratio, while the $\mathrm{C}$ image had brightened to the point where it is clearly brighter than the $\mathrm{B}$ component and rivals the A component in magnitude. A quantitative representation of these trends is provided by the light curves of the three brightest components, shown in Figure 4.

\section{THE A-B TIME DELAY}

Model predictions for the time delays between the six observed quasar images were reported in the work by Dahle et al. (2013). Being based on limited constraints from groundbased imaging (e.g., using only a single family of multiply lensed images, of the quasar itself), these predictions all have large uncertainty intervals of several hundred days.

The model prediction for the time delay between the $\mathrm{A}$ and $\mathrm{B}$ images reported by Dahle et al. (2013) is $\tau_{\mathrm{AB}}=112_{-158}^{+225}$ days, where positive values imply that image $\mathrm{A}$ is leading image $\mathrm{B}$.
The time delay between $\mathrm{A}$ and $\mathrm{C}$ is predicted to be $\tau_{\mathrm{CA}}=1256_{-411}^{+485}$ days. Given the much shorter predicted time delay between $\mathrm{A}$ and $\mathrm{B}$, we would expect the quasar variability light curves of these two images to overlap over a large fraction of the time interval covered by our photometric monitoring. Hence, we focus initially on determining the value of $\tau_{\mathrm{AB}}$.

While a large number of different methods for deriving the time delays of light curves of strong lenses have recently been proposed and tested (Liao et al. 2015), we focus here on two traditional methods for time delay measurements, detailed below. However, even prior to the implementation of these methods, an initial estimate of $\tau_{\mathrm{AB}}$ of $\sim 40-50$ days was made by shifting and visually matching the light curves. Finding a plausible match was facilitated by the obvious overall variability trends seen in the A and B light curves, showing continuous declines over $\sim 1.5$ year, followed by one observing season of nearly constant fluxes during 2014. Below, we use two different statistical methods to estimate $\tau_{\mathrm{AB}}$ in a more rigorous manner.

\section{1. $\chi^{2}$ Minimization}

This method is based on shifting the two observed light curves $A\left(t_{i}\right)$ and $B\left(t_{i}\right)$, cross-correlating each of them with a linearly interpolated light curve for the other image, $(b(t)$, and $a$ $(t)$, respectively), and finding the values of $\tau$ and the magnitude offset $\Delta m$ that minimize the statistic

$$
\begin{aligned}
\frac{\chi^{2}(\tau)}{N_{\mathrm{dof}}(\tau)}= & \frac{1}{2 N_{\mathrm{dof}}(\tau)} \sum_{i}^{N(\tau)} \frac{\left[A\left(t_{i}\right)-b\left(t_{i}-\tau\right)+\Delta m\right]^{2}}{\sigma_{A, i}^{2}+\sigma_{b, t}^{2}} \\
& +\frac{1}{2 N_{\mathrm{dof}}(\tau)} \sum_{i}^{N(\tau)} \frac{\left[a\left(t_{i}+\tau\right)-B\left(t_{i}\right)+\Delta m\right]^{2}}{\sigma_{a, t}^{2}+\sigma_{B, i}^{2}} .
\end{aligned}
$$

Here, $\sigma_{A, i}$ and $\sigma_{B, i}$ are errors in the observed magnitudes and $\sigma_{a, t}$ and $\sigma_{b, t}$ are errors in the interpolated magnitudes. We used data from all four seasons of systematic monitoring and assumed that $\Delta m_{\mathrm{AB}}$ did not change between seasons (such changes might be produced by microlensing). We did not interpolate between any set of points that spanned the gap between different seasons. The minimum value of $\frac{\chi^{2}(\tau)}{N_{\text {dof }}(\tau)}$ as a function of the time delay is shown in Figure 5. We find a minimum value for a time delay $\tau_{\mathrm{AB}}=42.0_{-0.1}^{+1.1}$ days and magnitude offset $\Delta m_{\mathrm{AB}}=0.345_{-0.005}^{+0.006}$ (with uncertainties corresponding to $\Delta \chi^{2}=4$ ).

\subsection{Dispersion Method}

A shortcoming of the $\chi^{2}$ minimization method is the assumption of linear flux variations between measured points in the light curve. A more statistically robust alternative method for time delay estimates was proposed by Pelt et al. (1994, 1996).

The method constructs a combined data set $C_{k}\left(t_{k}\right)$ from the two series $A_{i}$ (with $N_{A}$ measurements) and $B_{j}$ (with $N_{B}$ measurements), sampling the quasar light curve:

$$
C\left(t_{k}\right)= \begin{cases}A_{i} & t_{k}=t_{i} \\ B_{j}-\Delta m & t_{k}=t_{j}+\tau,\end{cases}
$$

where $k=1, \ldots, N$ and $N=N_{A}+N_{B}$. 
Table 1

$g$-band Photometry of the Three Brightest Quasar Images in SDSS J2222+2745

\begin{tabular}{|c|c|c|c|c|c|}
\hline MJD & Image A & Image B & Image $\mathrm{C}$ & Observatory & Detector \\
\hline 5122.200 & $21.100 \pm 0.030$ & $21.430 \pm 0.040$ & $21.510 \pm 0.050$ & SDSS & SDSS \\
\hline 5828.935 & $20.949 \pm 0.009$ & $21.422 \pm 0.009$ & $21.987 \pm 0.009$ & NOT & MOSCA \\
\hline 6182.963 & $21.092 \pm 0.009$ & $21.441 \pm 0.009$ & $22.111 \pm 0.009$ & NOT & MOSCA \\
\hline 6185.985 & $21.094 \pm 0.009$ & $21.465 \pm 0.009$ & $22.111 \pm 0.009$ & NOT & MOSCA \\
\hline 6244.907 & $21.170 \pm 0.014$ & $21.473 \pm 0.017$ & $22.145 \pm 0.030$ & NOT & ALFOSC \\
\hline 6244.911 & $21.148 \pm 0.014$ & $21.524 \pm 0.014$ & $22.083 \pm 0.019$ & NOT & ALFOSC \\
\hline 6244.915 & $21.155 \pm 0.014$ & $21.507 \pm 0.014$ & $22.100 \pm 0.021$ & NOT & ALFOSC \\
\hline 6266.907 & $21.187 \pm 0.014$ & $21.509 \pm 0.015$ & $22.101 \pm 0.023$ & NOT & ALFOSC \\
\hline 6266.911 & $21.214 \pm 0.014$ & $21.513 \pm 0.015$ & $22.080 \pm 0.022$ & NOT & ALFOSC \\
\hline 6270.830 & $21.218 \pm 0.015$ & $21.529 \pm 0.014$ & $22.122 \pm 0.021$ & NOT & ALFOSC \\
\hline 6270.834 & $21.213 \pm 0.015$ & $21.518 \pm 0.014$ & $22.089 \pm 0.020$ & NOT & ALFOSC \\
\hline 6270.838 & $21.224 \pm 0.015$ & $21.499 \pm 0.014$ & $22.070 \pm 0.020$ & NOT & ALFOSC \\
\hline 6291.811 & $21.301 \pm 0.019$ & $21.527 \pm 0.024$ & $22.120 \pm 0.037$ & NOT & ALFOSC \\
\hline 6291.815 & $21.242 \pm 0.015$ & $21.516 \pm 0.017$ & $22.168 \pm 0.028$ & NOT & ALFOSC \\
\hline 6291.819 & $21.288 \pm 0.014$ & $21.568 \pm 0.015$ & $22.201 \pm 0.024$ & NOT & ALFOSC \\
\hline 6430.182 & $21.396 \pm 0.015$ & $21.792 \pm 0.015$ & $22.189 \pm 0.019$ & NOT & ALFOSC \\
\hline 6430.189 & $21.441 \pm 0.015$ & $21.815 \pm 0.015$ & $22.243 \pm 0.019$ & NOT & ALFOSC \\
\hline 6430.197 & $21.413 \pm 0.015$ & $21.741 \pm 0.014$ & $22.198 \pm 0.019$ & NOT & ALFOSC \\
\hline 6446.168 & $21.363 \pm 0.015$ & $21.683 \pm 0.020$ & $22.191 \pm 0.028$ & NOT & ALFOSC \\
\hline 6446.182 & $21.327 \pm 0.014$ & $21.655 \pm 0.019$ & $22.111 \pm 0.025$ & NOT & ALFOSC \\
\hline 6454.178 & $21.430 \pm 0.014$ & $21.821 \pm 0.019$ & $22.234 \pm 0.026$ & NOT & ALFOSC \\
\hline 6454.182 & $21.443 \pm 0.015$ & $21.763 \pm 0.018$ & $22.261 \pm 0.026$ & NOT & ALFOSC \\
\hline 6454.186 & $21.382 \pm 0.014$ & $21.764 \pm 0.018$ & $22.233 \pm 0.025$ & NOT & ALFOSC \\
\hline 6457.183 & $21.388 \pm 0.007$ & $21.767 \pm 0.014$ & $22.254 \pm 0.015$ & NOT & ALFOSC \\
\hline 6457.190 & $21.401 \pm 0.011$ & $21.800 \pm 0.014$ & $22.260 \pm 0.016$ & NOT & ALFOSC \\
\hline 6457.198 & $21.373 \pm 0.011$ & $21.778 \pm 0.014$ & $22.260 \pm 0.016$ & NOT & ALFOSC \\
\hline 6477.090 & $21.452 \pm 0.015$ & $21.765 \pm 0.014$ & $22.207 \pm 0.019$ & NOT & ALFOSC \\
\hline 6477.097 & $21.455 \pm 0.014$ & $21.749 \pm 0.014$ & $22.251 \pm 0.018$ & NOT & ALFOSC \\
\hline 6477.105 & $21.463 \pm 0.014$ & $21.767 \pm 0.014$ & $22.295 \pm 0.018$ & NOT & ALFOSC \\
\hline 6488.130 & $21.444 \pm 0.010$ & $21.721 \pm 0.015$ & $22.220 \pm 0.016$ & NOT & ALFOSC \\
\hline 6488.137 & $21.472 \pm 0.010$ & $21.728 \pm 0.015$ & $22.249 \pm 0.016$ & NOT & ALFOSC \\
\hline 6488.145 & $21.443 \pm 0.010$ & $21.719 \pm 0.015$ & $22.227 \pm 0.016$ & NOT & ALFOSC \\
\hline 6507.150 & $21.470 \pm 0.016$ & $21.781 \pm 0.021$ & $22.289 \pm 0.030$ & NOT & ALFOSC \\
\hline 6507.158 & $21.455 \pm 0.016$ & $21.757 \pm 0.021$ & $22.265 \pm 0.030$ & NOT & ALFOSC \\
\hline 6507.165 & $21.445 \pm 0.015$ & $21.751 \pm 0.020$ & $22.283 \pm 0.030$ & NOT & ALFOSC \\
\hline 6518.141 & $21.451 \pm 0.015$ & $21.761 \pm 0.015$ & $22.288 \pm 0.020$ & NOT & ALFOSC \\
\hline 6518.148 & $21.471 \pm 0.014$ & $21.808 \pm 0.014$ & $22.302 \pm 0.018$ & NOT & ALFOSC \\
\hline 6518.156 & $21.459 \pm 0.013$ & $21.789 \pm 0.014$ & $22.281 \pm 0.017$ & NOT & ALFOSC \\
\hline 6533.039 & $21.475 \pm 0.015$ & $21.812 \pm 0.014$ & $22.213 \pm 0.017$ & NOT & ALFOSC \\
\hline 6533.050 & $21.502 \pm 0.015$ & $21.824 \pm 0.015$ & $22.262 \pm 0.019$ & NOT & ALFOSC \\
\hline 6533.058 & $21.507 \pm 0.015$ & $21.820 \pm 0.015$ & $22.235 \pm 0.019$ & NOT & ALFOSC \\
\hline 6533.065 & $21.466 \pm 0.014$ & $21.837 \pm 0.015$ & $22.285 \pm 0.022$ & NOT & ALFOSC \\
\hline 6543.103 & $21.497 \pm 0.014$ & $21.799 \pm 0.015$ & $22.212 \pm 0.020$ & NOT & ALFOSC \\
\hline 6543.110 & $21.496 \pm 0.014$ & $21.830 \pm 0.015$ & $22.208 \pm 0.019$ & NOT & ALFOSC \\
\hline 6543.118 & $21.501 \pm 0.014$ & $21.788 \pm 0.015$ & $22.187 \pm 0.019$ & NOT & ALFOSC \\
\hline 6549.047 & $21.528 \pm 0.015$ & $21.836 \pm 0.020$ & $22.138 \pm 0.026$ & NOT & ALFOSC \\
\hline 6549.054 & $21.461 \pm 0.014$ & $21.815 \pm 0.017$ & $22.220 \pm 0.023$ & NOT & ALFOSC \\
\hline 6557.850 & $21.555 \pm 0.014$ & $21.811 \pm 0.017$ & $22.197 \pm 0.023$ & NOT & ALFOSC \\
\hline 6557.854 & $21.552 \pm 0.014$ & $21.853 \pm 0.017$ & $22.137 \pm 0.022$ & NOT & ALFOSC \\
\hline 6557.858 & $21.523 \pm 0.015$ & $21.826 \pm 0.018$ & $22.172 \pm 0.024$ & NOT & ALFOSC \\
\hline 6565.970 & $21.573 \pm 0.012$ & $21.800 \pm 0.015$ & $22.169 \pm 0.014$ & NOT & ALFOSC \\
\hline 6565.977 & $21.559 \pm 0.013$ & $21.829 \pm 0.014$ & $22.196 \pm 0.015$ & NOT & ALFOSC \\
\hline 6565.985 & $21.531 \pm 0.011$ & $21.811 \pm 0.015$ & $22.137 \pm 0.014$ & NOT & ALFOSC \\
\hline 6573.944 & $21.579 \pm 0.010$ & $21.810 \pm 0.015$ & $22.162 \pm 0.014$ & NOT & ALFOSC \\
\hline 6573.951 & $21.560 \pm 0.010$ & $21.806 \pm 0.015$ & $22.182 \pm 0.014$ & NOT & ALFOSC \\
\hline 6573.958 & $21.579 \pm 0.009$ & $21.853 \pm 0.015$ & $22.170 \pm 0.014$ & NOT & ALFOSC \\
\hline 6578.051 & $21.520 \pm 0.018$ & $21.737 \pm 0.023$ & $22.088 \pm 0.031$ & NOT & ALFOSC \\
\hline 6578.055 & $21.542 \pm 0.020$ & $21.806 \pm 0.025$ & $22.078 \pm 0.031$ & NOT & ALFOSC \\
\hline 6578.059 & $21.577 \pm 0.017$ & $21.841 \pm 0.022$ & $22.149 \pm 0.027$ & NOT & ALFOSC \\
\hline 6602.891 & $21.634 \pm 0.015$ & $21.868 \pm 0.014$ & $22.135 \pm 0.015$ & NOT & ALFOSC \\
\hline 6602.899 & $21.621 \pm 0.014$ & $21.889 \pm 0.015$ & $22.160 \pm 0.018$ & NOT & ALFOSC \\
\hline 6602.906 & $21.585 \pm 0.011$ & $21.862 \pm 0.015$ & $22.156 \pm 0.014$ & NOT & ALFOSC \\
\hline 6618.884 & $21.650 \pm 0.014$ & $21.899 \pm 0.015$ & $22.179 \pm 0.018$ & NOT & ALFOSC \\
\hline 6618.888 & $21.638 \pm 0.014$ & $21.880 \pm 0.015$ & $22.163 \pm 0.017$ & NOT & ALFOSC \\
\hline
\end{tabular}


Table 1

(Continued)

\begin{tabular}{|c|c|c|c|c|c|}
\hline MJD & Image A & Image B & Image $\mathrm{C}$ & Observatory & Detector \\
\hline 6618.891 & $21.602 \pm 0.014$ & $21.903 \pm 0.016$ & $22.139 \pm 0.020$ & NOT & ALFOSC \\
\hline 6788.186 & $21.572 \pm 0.015$ & $21.916 \pm 0.019$ & $22.160 \pm 0.022$ & NOT & ALFOSC \\
\hline 6788.193 & $21.597 \pm 0.015$ & $22.003 \pm 0.020$ & $22.133 \pm 0.022$ & NOT & ALFOSC \\
\hline 6815.173 & $21.556 \pm 0.015$ & $21.942 \pm 0.015$ & $22.113 \pm 0.016$ & NOT & ALFOSC \\
\hline 6815.181 & $21.570 \pm 0.015$ & $21.960 \pm 0.015$ & $22.112 \pm 0.016$ & NOT & ALFOSC \\
\hline 6815.188 & $21.565 \pm 0.015$ & $21.942 \pm 0.015$ & $22.101 \pm 0.016$ & NOT & ALFOSC \\
\hline 6835.176 & $21.614 \pm 0.014$ & $21.927 \pm 0.014$ & $22.076 \pm 0.015$ & NOT & ALFOSC \\
\hline 6835.184 & $21.584 \pm 0.015$ & $21.914 \pm 0.014$ & $22.080 \pm 0.015$ & NOT & ALFOSC \\
\hline 6835.191 & $21.571 \pm 0.015$ & $21.924 \pm 0.015$ & $22.117 \pm 0.016$ & NOT & ALFOSC \\
\hline 6847.162 & $21.584 \pm 0.014$ & $21.901 \pm 0.015$ & $22.022 \pm 0.016$ & NOT & ALFOSC \\
\hline 6847.169 & $21.574 \pm 0.014$ & $21.916 \pm 0.015$ & $22.062 \pm 0.016$ & NOT & ALFOSC \\
\hline 6847.179 & $21.589 \pm 0.014$ & $21.908 \pm 0.016$ & $22.060 \pm 0.016$ & NOT & ALFOSC \\
\hline 6864.105 & $21.584 \pm 0.015$ & $21.959 \pm 0.015$ & $22.017 \pm 0.015$ & NOT & ALFOSC \\
\hline 6874.056 & $21.610 \pm 0.014$ & $21.914 \pm 0.015$ & $21.950 \pm 0.015$ & NOT & ALFOSC \\
\hline 6874.063 & $21.612 \pm 0.015$ & $21.934 \pm 0.014$ & $21.952 \pm 0.014$ & NOT & ALFOSC \\
\hline 6894.020 & $21.610 \pm 0.012$ & $21.937 \pm 0.014$ & $21.963 \pm 0.014$ & NOT & ALFOSC \\
\hline 6894.028 & $21.592 \pm 0.014$ & $21.902 \pm 0.014$ & $21.903 \pm 0.014$ & NOT & ALFOSC \\
\hline 6894.035 & $21.599 \pm 0.015$ & $21.892 \pm 0.014$ & $21.943 \pm 0.014$ & NOT & ALFOSC \\
\hline 6918.096 & $21.565 \pm 0.014$ & $21.956 \pm 0.018$ & $21.915 \pm 0.017$ & NOT & ALFOSC \\
\hline 6928.011 & $21.619 \pm 0.015$ & $21.952 \pm 0.014$ & $21.956 \pm 0.014$ & NOT & ALFOSC \\
\hline 6928.019 & $21.620 \pm 0.014$ & $21.987 \pm 0.015$ & $21.944 \pm 0.015$ & NOT & ALFOSC \\
\hline 6928.026 & $21.625 \pm 0.013$ & $21.942 \pm 0.014$ & $21.959 \pm 0.014$ & NOT & ALFOSC \\
\hline 6944.979 & $21.600 \pm 0.014$ & $21.933 \pm 0.015$ & $21.923 \pm 0.014$ & NOT & ALFOSC \\
\hline 6944.986 & $21.628 \pm 0.015$ & $21.921 \pm 0.014$ & $21.960 \pm 0.014$ & NOT & ALFOSC \\
\hline 6944.993 & $21.625 \pm 0.014$ & $21.933 \pm 0.015$ & $21.900 \pm 0.015$ & NOT & ALFOSC \\
\hline 6973.943 & $21.629 \pm 0.018$ & $21.931 \pm 0.024$ & $21.699 \pm 0.019$ & NOT & ALFOSC \\
\hline 6973.950 & $21.627 \pm 0.018$ & $21.920 \pm 0.024$ & $21.726 \pm 0.019$ & NOT & ALFOSC \\
\hline 6973.958 & $21.524 \pm 0.019$ & $21.952 \pm 0.027$ & $21.709 \pm 0.021$ & NOT & ALFOSC \\
\hline 6986.896 & $21.643 \pm 0.015$ & $21.883 \pm 0.018$ & $21.654 \pm 0.015$ & NOT & ALFOSC \\
\hline 6986.903 & $21.642 \pm 0.014$ & $21.920 \pm 0.017$ & $21.683 \pm 0.014$ & NOT & ALFOSC \\
\hline 6986.911 & $21.623 \pm 0.014$ & $21.918 \pm 0.017$ & $21.668 \pm 0.014$ & NOT & ALFOSC \\
\hline 7141.226 & $21.651 \pm 0.031$ & $22.003 \pm 0.039$ & $21.788 \pm 0.034$ & NOT & ALFOSC \\
\hline 7143.207 & $21.696 \pm 0.024$ & $22.050 \pm 0.030$ & $21.772 \pm 0.024$ & NOT & ALFOSC \\
\hline 7143.211 & $21.744 \pm 0.020$ & $22.067 \pm 0.027$ & $21.809 \pm 0.022$ & NOT & ALFOSC \\
\hline 7143.214 & $21.696 \pm 0.018$ & $21.966 \pm 0.026$ & $21.742 \pm 0.019$ & NOT & ALFOSC \\
\hline 7168.539 & $21.789 \pm 0.009$ & $22.031 \pm 0.009$ & $21.836 \pm 0.009$ & Gemini & GMOS \\
\hline 7169.188 & $21.750 \pm 0.014$ & $21.986 \pm 0.016$ & $21.822 \pm 0.014$ & NOT & ALFOSC \\
\hline 7169.195 & $21.775 \pm 0.014$ & $22.061 \pm 0.016$ & $21.824 \pm 0.014$ & NOT & ALFOSC \\
\hline 7169.203 & $21.800 \pm 0.014$ & $22.014 \pm 0.017$ & $21.826 \pm 0.014$ & NOT & ALFOSC \\
\hline 7169.518 & $21.787 \pm 0.009$ & $22.025 \pm 0.009$ & $21.830 \pm 0.009$ & Gemini & GMOS \\
\hline 7191.154 & $21.771 \pm 0.014$ & $22.044 \pm 0.015$ & $21.818 \pm 0.014$ & NOT & ALFOSC \\
\hline 7191.161 & $21.777 \pm 0.014$ & $22.029 \pm 0.016$ & $21.822 \pm 0.014$ & NOT & ALFOSC \\
\hline 7191.168 & $21.783 \pm 0.014$ & $21.996 \pm 0.016$ & $21.857 \pm 0.014$ & NOT & ALFOSC \\
\hline 7196.183 & $21.784 \pm 0.015$ & $22.052 \pm 0.014$ & $21.828 \pm 0.014$ & NOT & ALFOSC \\
\hline 7196.190 & $21.809 \pm 0.014$ & $22.039 \pm 0.015$ & $21.817 \pm 0.014$ & NOT & ALFOSC \\
\hline 7196.197 & $21.807 \pm 0.014$ & $22.059 \pm 0.015$ & $21.819 \pm 0.014$ & NOT & ALFOSC \\
\hline 7218.080 & $21.794 \pm 0.016$ & $22.110 \pm 0.022$ & $21.740 \pm 0.015$ & NOT & ALFOSC \\
\hline 7218.087 & $21.763 \pm 0.016$ & $22.054 \pm 0.021$ & $21.731 \pm 0.016$ & NOT & ALFOSC \\
\hline 7228.476 & $21.791 \pm 0.009$ & $22.096 \pm 0.009$ & $21.793 \pm 0.009$ & Gemini & GMOS \\
\hline 7256.088 & $21.687 \pm 0.014$ & $22.066 \pm 0.016$ & $21.748 \pm 0.014$ & NOT & ALFOSC \\
\hline 7258.077 & $21.725 \pm 0.014$ & $22.079 \pm 0.014$ & $21.746 \pm 0.014$ & NOT & ALFOSC \\
\hline 7258.084 & $21.697 \pm 0.015$ & $22.121 \pm 0.015$ & $21.723 \pm 0.015$ & NOT & ALFOSC \\
\hline 7258.091 & $21.696 \pm 0.015$ & $22.066 \pm 0.015$ & $21.733 \pm 0.015$ & NOT & ALFOSC \\
\hline 7260.148 & $21.691 \pm 0.014$ & $22.087 \pm 0.017$ & $21.763 \pm 0.014$ & NOT & ALFOSC \\
\hline
\end{tabular}

Note. The Modified Julian Days (MJD) column gives the date of the observation relative to MJD $=50000$. 

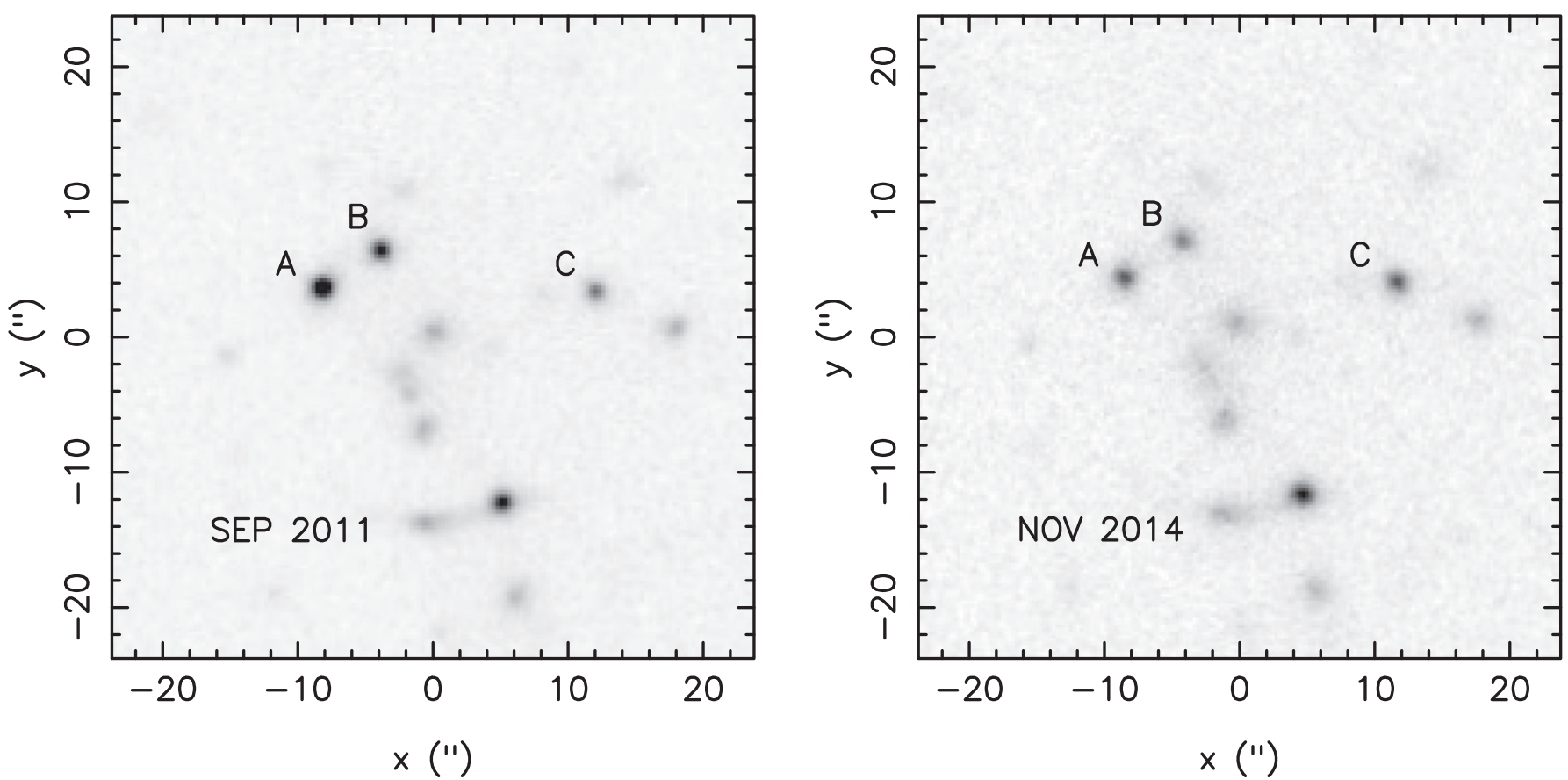

Figure 3. Left panel: MOSCA image from 2011 September. Right panel: ALFOSC image from 2014 November, illustrating the flux variation of the C image relative to the A and B images of the quasar during our monitoring of SDSS J2222+2745.

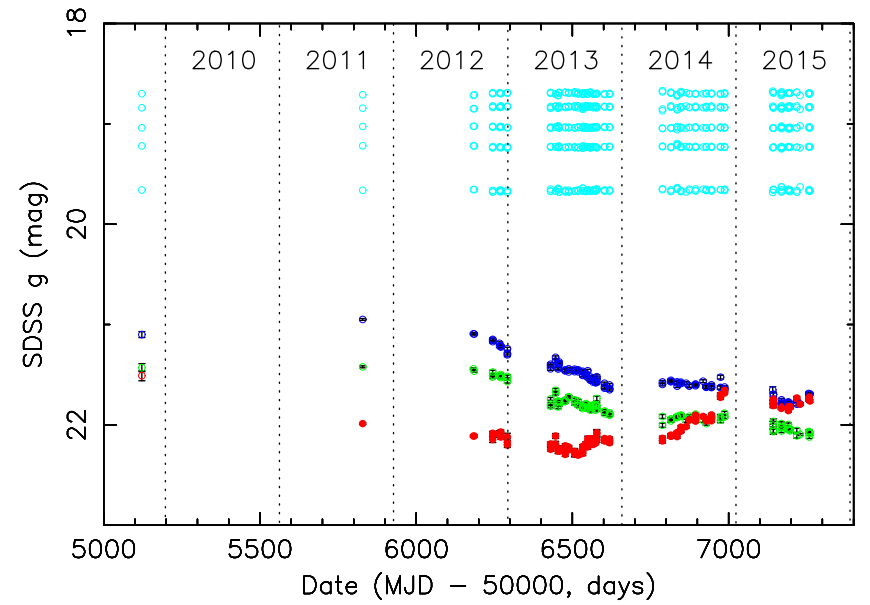

Figure 4. Light curves of the quasar images A (blue symbols), B (green), and C (red). The light blue points in the upper part of the image represent the photometric measurements of the comparison stars S1-S5.

From this combined data set, the dispersion spectrum is defined by

$$
D^{2}(\tau)=\min _{\Delta m} \frac{\sum_{k=1}^{N-1} S_{k} W_{k} G_{k}\left(C_{k+1}-C_{k}\right)^{2}}{2 \sum_{k=1}^{N-1} S_{k} W_{k} G_{k}},
$$

where $G_{k}=1$ only if the measurements are from different images and $G_{k}=0$ otherwise, $W_{k}=\left(\sigma_{k}^{2}+\sigma_{k+1}^{2}\right)^{-1}$ are statistical weights, and

$$
S_{k}= \begin{cases}1 & \left|t_{k+1}-t_{k}\right| \leqslant \delta \\ 0 & \left|t_{k+1}-t_{k}\right|>\delta .\end{cases}
$$

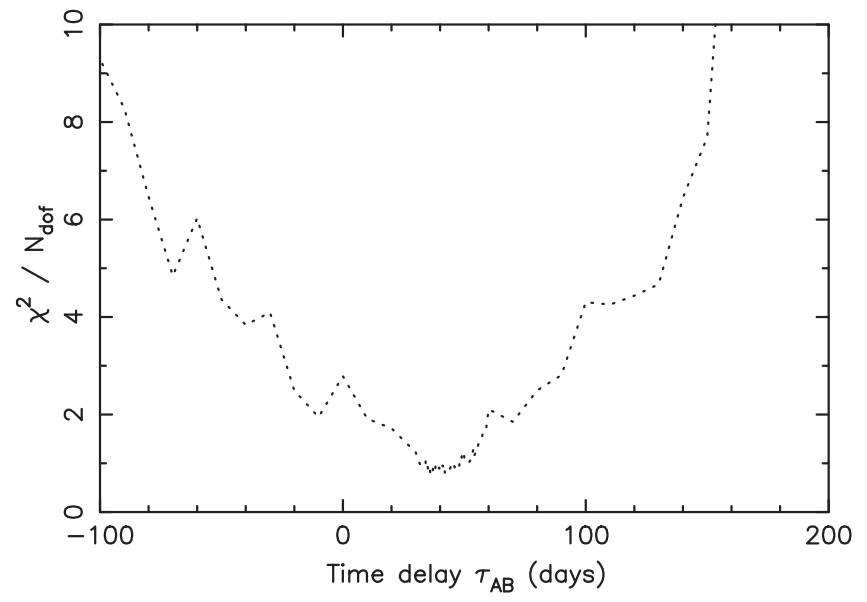

Figure 5. Result of a $\chi^{2}$ minimization obtained by shifting and matching the observed light curves for A and B measured during the period 2012 September-2015 August.

We used $\delta=25$ days as the decorrelation timescale, producing the dispersion spectrum shown in Figure 6. The minimum value is reached for $\tau_{\mathrm{AB}}=47.7 \pm 6.0$ days and $\Delta m_{\mathrm{AB}}=0.340 \pm 0.007$ (95\% confidence intervals). The result has little sensitivity to the choice of $\delta$; choosing a number twice or half the value quoted above changes the best fit values by a few percent, well within the uncertainty intervals quoted below.

To estimate the uncertainties, we used a bootstrap procedure. The light curve was smoothed using a running 9-point median filter and the residuals of our data points were calculated with respect to the smoothed light curve. A set of 1000 simulated light curves were then generated by randomly re-shuffling the residuals, and the errors were estimated from the spread of values of $\tau_{\mathrm{AB}}$ from the 1000 dispersion spectra. A combined $\mathrm{A}+\mathrm{B}$ light curve $C\left(t_{k}\right)$ is represented by the union of the red and blue points plotted in Figure 9. In this figure, the observed 


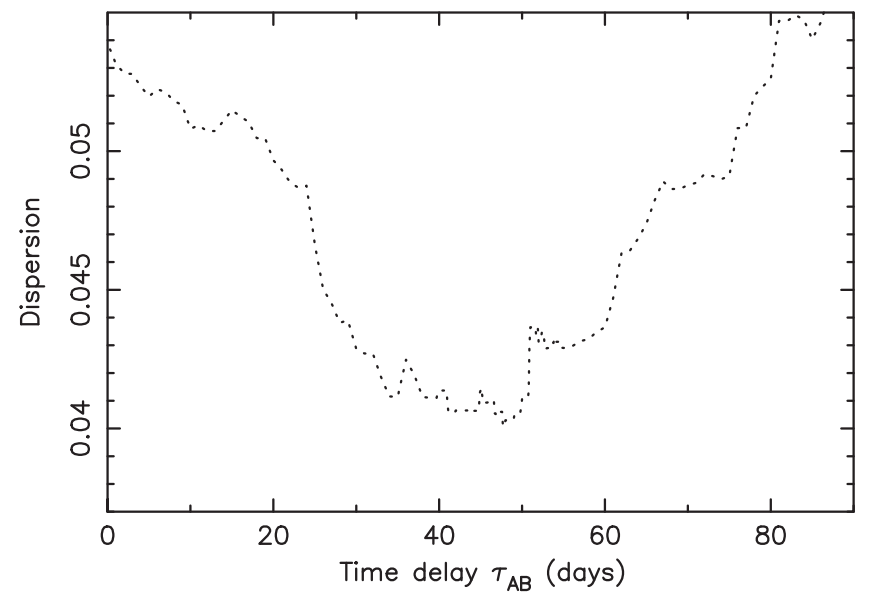

Figure 6. Dispersion calculated as a function of the shift $\tau_{\mathrm{AB}}$ between the two light curves.

light curve of the $\mathrm{B}$ component has been shifted by the $\tau_{\mathrm{AB}}$ and $\Delta m_{\mathrm{AB}}$ values derived using the dispersion method.

\section{THE A-C TIME DELAY}

Having estimated $\tau_{\mathrm{AB}}$, we next consider the time delay between the combined $\mathrm{AB}$ light curve and image $\mathrm{C}$. For this purpose, we also include the 2009 photometry from SDSS DR8. All acceptable lens models of this system predict that image $\mathrm{C}$ leads all the other images, so we do not consider time delays $\tau_{\mathrm{CA}}<0$. A $\chi^{2}$ minimization using the procedure in Section 3.1 yields a minimum at time delay $\tau_{\mathrm{CA}}=717.4 \pm 0.5$ days, with uncertainties corresponding to $\Delta \chi^{2}=4$. The magnitude offset is constrained as $\Delta m_{\mathrm{AC}}=0.504 \pm 0.013$. The minimum value of $\frac{\chi^{2}(\tau)}{N_{\text {dof }}(\tau)}$ as a function of the time delay is shown in Figure 7. No meaningful calculation could be done for time delay values greater than 830 days, since there would so far be minimal or no overlap between the monitoring light curves for such long time delays.

We also estimated $\tau_{\mathrm{CA}}$ using the dispersion method described in Section 3.2. This produced the dispersion spectrum shown in Figure 8, yielding a best-fit time delay of $\tau_{\mathrm{CA}}=722 \pm 24$ days and magnitude offset $\Delta m_{\mathrm{AC}}=$ $0.483 \pm 0.012$ (95\% confidence intervals). These values are consistent with the values produced by the $\chi^{2}$ minimization method. Since the dispersion method does not rely on assumptions about the quasar flux variation between monitoring epochs, we consider the values and uncertainties produced by this method to be more reliable than those produced by interpolation and $\chi^{2}$ minimization. Hence, we adopt the time delay $\tau_{\mathrm{CA}}=722 \pm 24$ days in our further discussion.

As shown in Figure 9, the best-fit value derived for $\tau_{\mathrm{CA}}$ makes very distinctive predictions for the photometric behavior of images A and B during the 2015 and 2016 monitoring seasons: the A and B light curves are both predicted to reach a sharp minimum, spaced by their 47.7-day time delay, in mid2015. This minimum corresponds to the lowest luminosity state of the quasar observed during the $\sim 8$ years of intrinsic quasar brightness variations spanned by all available photometric data. Our most recent data points, from 2015 August 22, 24, and 26 UT (see Table 1), show an increase in the flux of image A by $\sim 0.1$ mag after passing the global minimum in the intrinsic quasar light curve, exactly as predicted by the best-fit $\tau_{\mathrm{CA}}$

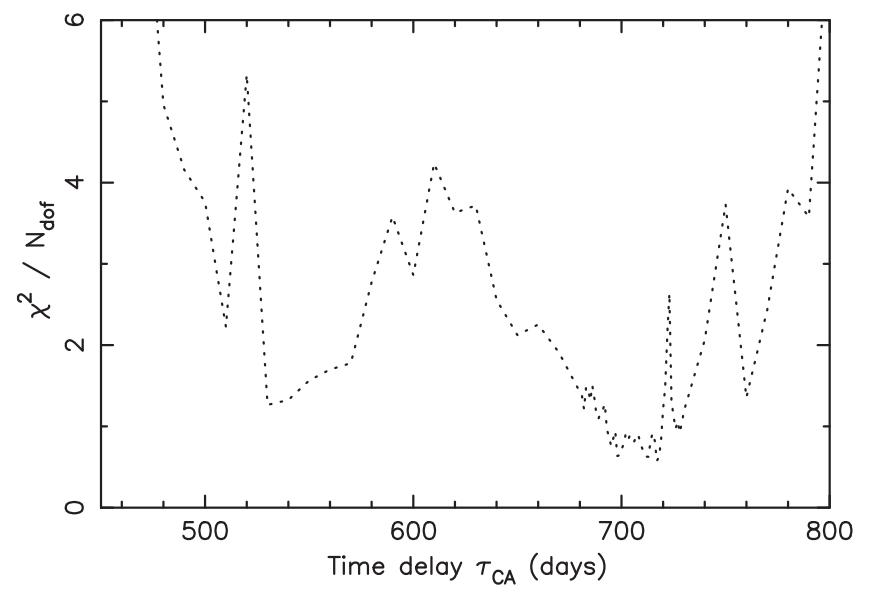

Figure 7. Result of a $\chi^{2}$ minimization obtained by shifting and matching the observed light curve of $\mathrm{C}$ to the combined $\mathrm{A}+\mathrm{B}$ light curve.

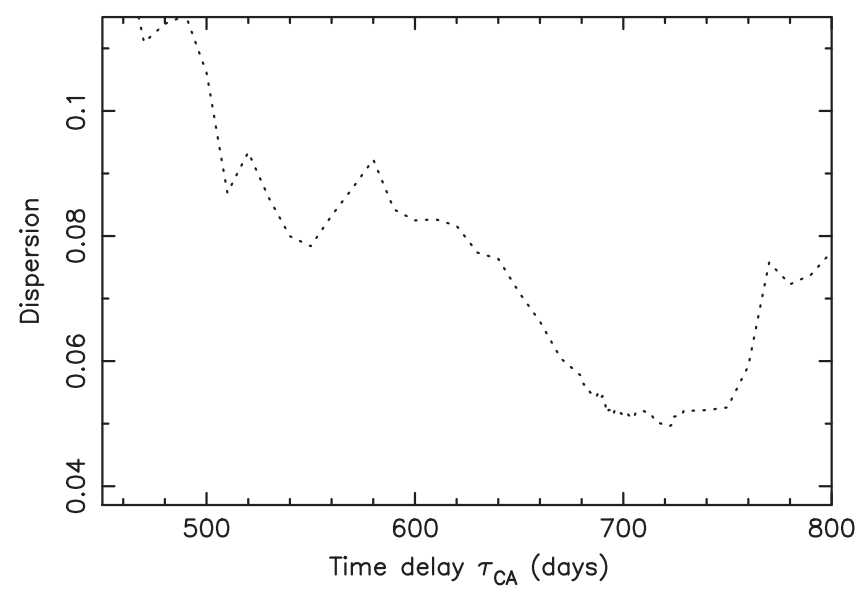

Figure 8. Dispersion calculated as a function of the shift $\tau_{\mathrm{CA}}$ between the observed light curve of image $\mathrm{C}$ and the combined $\mathrm{A}+\mathrm{B}$ light curve.

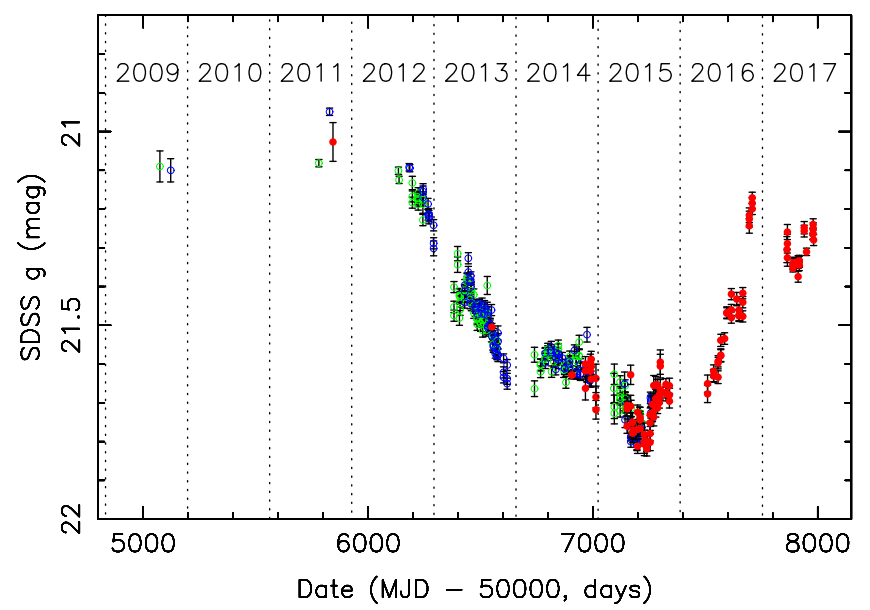

Figure 9. Combined A (open blue circles) + B (open green circles) light curve, together with the $\mathrm{C}$ (filled red circles) light curve, the latter shifted by $\tau_{\mathrm{CA}}=722$ days and $\Delta m_{\mathrm{AC}}=0.483$. The dates and magnitude values correspond to the light curve of the A image.

value. Continued monitoring of images $\mathrm{A}$ and $\mathrm{B}$, tracking their predicted further brightening during the fall of 2015, should substantially reduce the size of the uncertainty intervals of both $\tau_{\mathrm{AB}}$ and $\tau_{\mathrm{CA}}$. 
The fluxes of A and B are predicted to further increase by $>0.5$ mag during 2016. Observing these predicted variations of image A and B through the end of 2016 will strictly exclude any alternative time delay values in the broad interval $830 \lesssim \tau_{\mathrm{CA}} \lesssim 2100$ days. We note that the upper limit of this interval extends well beyond the range of time delays allowed by the lens model of Dahle et al. (2013).

\section{SUMMARY AND DISCUSSION}

Predictions from the lens model presented by Dahle et al. (2013) indicate that three independent time delays $\tau_{\mathrm{AB}}, \tau_{\mathrm{ED}}$, and $\tau_{\mathrm{FE}}$ in the system are all of the order of 100 days. The predicted time delays between image $\mathrm{C}$ and the pair (A-B) and between (A-B) and the central images (D-E-F) are of the order of 1300 days and 700-1000 days, respectively (with $\sim 40 \%$ uncertainty).

We have measured $\tau_{\mathrm{AB}}$ using two different methods and find results that are consistent at the $2 \sigma$ level. However, unlike the $\chi^{2}$ minimization method, the dispersion method does not make any assumptions about the photometric behavior of the quasar images during the $\sim 14$ day sampling intervals, and we therefore adopt the dispersion method value of $\tau_{\mathrm{AB}}=47.7 \pm 6.0$ days $(95 \%$ confidence interval) as the most robust measurement. This is consistent with the model predicted value of $\tau_{\mathrm{AB}}=112_{-158}^{+225}$ days. The measured magnitude difference $\Delta m_{\mathrm{AB}}=0.340 \pm 0.007$ is also consistent with the ratio of the model-predicted magnifications of $\mu_{\mathrm{A}}=5.4_{-1.6}^{+4.6}$ and $\mu_{\mathrm{B}}=4.7_{-1.6}^{+3.4}$ (Dahle et al. 2013).

The measured $\tau_{\mathrm{AB}}$ time delay is very close to 1.5 synodic months, implying that it is possible to construct a continuous (apart from seasonal gaps) quasar light curve from the combination of data points from image A and image B which will be minimally affected by poor-signal-to-noise ratio measurements caused by bright moonlight.

From our systematic monitoring campaign, there is so far only a year of overlap between the intrinsic quasar light curve derived from image $\mathrm{C}$ and those from $\mathrm{A}$ and $\mathrm{B}$. However, the strong amplitude of the intrinsic brightness fluctuations of the quasar, the presence of earlier data points from 2009 and 2011, and the excellent match of the sharp flux minima observed in image C in 2013 and in images A and B in 2015 enable us to measure the $\tau_{\mathrm{CA}}$ time delay from the data set currently in hand.

The best-fit value of $\tau_{\mathrm{CA}}=722 \pm 24$ days is in slight tension with the model-predicted value of $\tau_{\mathrm{CA}}=1256_{-411}^{+485}$ days of Dahle et al. (2013). We note that ongoing work, refining the lens model by incorporating additional constraints from newly identified multiply imaged source galaxies in this lens system, produces systematically lower model-predicted values for $\tau_{\mathrm{CA}}$. The measured magnitude difference $\Delta m_{\mathrm{AC}}=0.483 \pm 0.012$ is consistent within the errors with the ratio of the modelpredicted magnifications of $\mu_{\mathrm{A}}=5.4_{-1.6}^{+4.6}$ and $\mu_{\mathrm{C}}=2.4_{-0.3}^{+1.1}$ (Dahle et al. 2013).

Our prediction of the timing of the expected $0.5-0.6$ mag rise in images $\mathrm{A}$ and $\mathrm{B}$ can be used to plan a more intensive campaign to monitor photometric and spectroscopic changes in the quasar during this phase in 2016. This would, e.g., allow a reverberation mapping study of the $z=2.82$ quasar.

A subsequent corresponding brightness increase should occur for the three fainter images around 2018, given the Dahle et al. (2013) prediction for the time delays between the
(A-B) pair and the (D-F) trio. During 2015, these fainter images should be entering the decline phase seen in images A and B in 2012-2013, before reaching the plateau seen in 2014. Provided that even rough estimates of the time delays can be made from observations in the decline and subsequent plateau phase, a targeted campaign at the predicted time of the subsequent rise could provide accurate time delay estimates for the three demagnified images in the cluster center.

We have recently obtained 5-band $H S T$ optical/NIR imaging (GO-13337; PI: Sharon) of SDSS J2222+2745 that confirms the existence of the sixth quasar image and reveals additional multiply lensed background galaxies. Additional constraints from the positions and redshifts of these, together with the measurements reported here for $\tau_{\mathrm{AB}}$ and $\tau_{\mathrm{CA}}$, will allow us to refine the lens model of this system and provide updated predictions for the unmeasured time delays. The HST data will also be crucial for modeling the brightness distribution of the brightest galaxies in the cluster core and removing their contribution to the measured flux of images D-F in ground-based photometric monitoring data.

To summarize: the steep quasar brightness fluctuations evident from the light curve of the leading $\mathrm{C}$ image provide strong constraining power to measurements of the time delays between all six known images in this lens system. The range of clustercentric radii over which such measurements are possible is truly unique for this system. The next $2-3$ observing seasons will be instrumental in pinning down these time delays, enabling a wide range of physical studies of both the cluster lens and the quasar source.

H.D. acknowledges support from the Research Council of Norway. M.B.B. acknowledges support from the NSF through grant AST-1009012. We acknowledge use of the new Gemini Fast Turnaround Program and the Fast-Track Service mode at NOT, which both provided important data for this paper. We thank the staffs of NOT and Gemini for their flexibility in supporting the execution of our monitoring campaign. The authors wish to recognize and acknowledge the very significant cultural role and reverence that the summit of Mauna Kea has always had within the indigenous Hawaiian community. We are most fortunate to have the opportunity to conduct observations from this mountain.

\section{REFERENCES}

Bar-Kana, R. 1996, ApJ, 468, 17

Blandford, R. D., \& McKee, C. F. 1982, ApJ, 255, 419

Dahle, H., Gladders, M. D., Sharon, K., et al. 2013, ApJ, 773, 146

Fohlmeister, J., Kochanek, C. S., Falco, E. E., et al. 2013, ApJ, 764, 186

Fohlmeister, J., Kochanek, C. S., Falco, E. E., Morgan, C. W., \& Wambsganss, J. 2008, ApJ, 676, 761

Inada, N., Oguri, M., Morokuma, T., et al. 2006, ApJL, 653, L97

Inada, N., Oguri, M., Pindor, B., et al. 2003, Natur, 426, 810

Liao, K., Treu, T., Marshall, P., et al. 2015, ApJ, 800, 11

Linder, E. V. 2011, PhRvD, 84, 123529

Misawa, T., Inada, N., Oguri, M., et al. 2014, ApJL, 794, LL20

Moustakas, L. A., Brownstein, J., Fadely, R., et al. 2012, in American Astronomical Society Meeting Abstracts, 219, 146.01

Oguri, M. 2010, PASJ, 62, 1017

Oguri, M., Schrabback, T., Jullo, E., et al. 2013, MNRAS, 429, 482

Pelt, J., Hoff, W., Kayser, R., Refsdal, S., \& Schramm, T. 1994, A\&A, 286, 775

Pelt, J., Kayser, R., Refsdal, S., \& Schramm, T. 1996, A\&A, 305, 97

Peterson, B. M. 1993, PASP, 105, 247 
Peterson, B. M. 2014, SSRv, 183, 253

Refsdal, S. 1964, MNRAS, 128, 307

Refsdal, S. 1966, MNRAS, 132, 101

Sereno, M., \& Paraficz, D. 2014, MNRAS, 437, 600
Sharon, K., Ofek, E. O., Smith, G. P., et al. 2005, ApJL, 629, L73

Treu, T., Marshall, P. J., Cyr-Racine, F.-Y., et al. 2013, arXiv:1306.1272

Vanden Berk, D. E., Wilhite, B. C., Kron, R. G., et al. 2004, ApJ, 601, 692

Wambsganss, J., Bode, P., \& Ostriker, J. P. 2005, ApJL, 635, L1 